\title{
Revue systématique des études d'intervention alliant promotion de la diététique et de l'activité physique dans les écoles en Europe
}

\author{
C. Carette \\ (C) Springer-Verlag France 2011
}

Les auteurs de cette revue ont porté leur attention sur les études d'intervention qui comprenaient à la fois une éducation alimentaire mais aussi la promotion de l'activité physique. Les études qui ne comprenaient que l'un ou l'autre de ces volets éducatifs ont été exclues. Ils ont également choisi de se concentrer sur les études européennes uniquement.

La méthode de sélection des articles est une méthode de recherche de publications électroniques et ensuite de premier tri basé sur le titre et l'abstract (environ 9000 articles identifiés au départ en entrant les mots clés dans les bases de données) [1]. Les études qui ne comportaient pas de données anthropométriques et comportementales ou qui n'étaient pas basées sur une prise en charge à l'école ont été exclues. La plupart de ces études d'intervention ont été réalisées aux États-Unis ou en Australie et, finalement, les auteurs ont identifié 11 études européennes à inclure. Aucune de ces études n'avait été conduite en France. Parmi ces 11 études, six portaient sur les jeunes enfants et cinq sur les adolescents. En raison de l'hétérogénéité de ces études, une méta-analyse n'a pas pu être conduite. Il s'agit donc d'une revue de la littérature systématique et descriptive.

Les auteurs montrent qu'il existe une plus faible augmentation de l'IMC chez les adolescentes dans deux études d'intervention [2,3] qui comprenaient une approche à la fois éducationnelle faisant la promotion de l'activité physique et d'une meilleure hygiène alimentaire mais aussi environnementale (ces deux études étaient randomisées avec comparaison à une population d'adolescentes témoins). En effet, il apparaît que, pour obtenir un effet durable concernant l'IMC (à deux et dix ans respectivement dans ces études), il faut associer des cours collectifs et individuels à des changements de pratique au sein des établissements scolaires, avec modification des menus à la cantine, de l'accès aux aliments dans l'école et augmentation de l'activité physique scolaire et extrascolaire. Dans ces deux études, seules les jeunes filles semblent bénéficier de cette approche éducationnelle et environnementale en milieu scolaire. Une tentative d'explication est proposée, car dans une étude, il a été mesuré chez les garçons une moins bonne adhésion aux mesures diététiques alors que l'activité physique était augmentée comme chez les filles.

Chez les enfants plus jeunes (6-12 ans), les auteurs estiment que les études ont un trop faible niveau de preuve, ce qui ne permet pas de conclure sur l'intérêt de ces interventions globales en Europe dans cette tranche d'âge.

En conclusion, cette revue descriptive et systématique des études d'intervention en milieu scolaire confirme qu'il est important d'associer des mesures de changement de l'environnement obésogène des enfants aux classiques cours collectifs ou individuels. En effet, c'est par cette prise en charge globale environnementale et éducationnelle prenant en compte la diététique et l'activité physique que deux études ont pu montrer un effet sur l'évolution de l'IMC de jeunes adolescentes.

\section{Références}

1. De Bourdeaudhuij I (2011) School-based interventions promoting both physical activity and healthy eating in Europe: a systematic review within the HOPE project. Obes Rev 12(3):205-16

2. Haerens L (2006) Body mass effect of a physical activity and healthy eating intervention in middle schools. Obesity 14:847-54

3. Singh (2007) Short-term effects of school-based weight gain prevention among adolescents. Arch Pediatr Adolesc Med 161:565-71

\footnotetext{
C. Carette $(\bowtie)$

« Expression génique, développement et maladies » (EGDM), équipe 26/Inserm U1016/CNRS UMR 8104/université Paris-Descartes, institut Cochin, département génétique et développement, 24, rue du Faubourg-Saint-Jacques, F-75014 Paris, France

e-mail : claire.carette@inserm.fr
} 